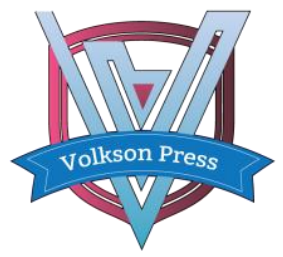

\title{
STATISTICS, MONITORING AND EARLY WARNING OF AGRICULTURAL PRODUCTS CIRCULATION AND CONSUMPTION PRICE IN GUANGDONG PROVINCE OF CHINA
}

\author{
Li Dan-xia' ${ }^{1,2 *}$, Wan Zhong1,2 \\ ${ }^{1}$ Guangdong Academy of agricultural Sciences, Institute of Agricultural and Rural Economic Development, Guangzhou 510640 \\ ${ }^{2}$ Southern China Key Laboratory of urban agriculture, Ministry of Agriculture, Guangzhou 510640 \\ *Corresponding Author's E-mail: $\underline{625005883 @ q q . c o m}$
}

This is an open access article distributed under the Creative Commons Attribution License, which permits unrestricted use, distribution, and reproduction in any medium, provided the original work is properly cited.

\section{ARTICLE DETAILS}

\section{Article History:}

Received 12 March 2018 Accepted 12 April 2018 Available online 15 May 2018

\begin{abstract}
In this study, the monitoring index database of agricultural products price in Guangdong province of China was constructed. It monitored 2 categories including commodity retail price index, wholesale and retail price, 6 varieties and 11 fine items including agricultural products circulation index; The consumer price index of agricultural products consumption index 1 categories, 4 varieties of indicators. The price circulation and consumption data of 8 agricultural products from 7 units including national bureau of statistics, Guangdong bureau of statistics, Guangdong development and reform commission and price monitoring center, Guangdong department of agriculture, Guangdong grain bureau, Guangzhou Jiangnan fruit and vegetable market, Zhengzhou Hualiang technology co., ltd. were collected and monitored.
\end{abstract}

Based on the statistics of agricultural products circulation, consumption indicators and other data, this study chose to build a general model group for agricultural products monitoring, early warning, using the combination of exponential smoothing model, and ARIMA model, the warning value of circulation and consumption of agricultural products is set based on the historical data and forecast value of circulation and consumption of agricultural products.

\section{KEYWORDS}

Agricultural product circulation price statistics, Agricultural product consumption price statistics, Agricultural product price monitoring, Agricultural product price warning.

\section{INTRODUCTION}

Guangdong province, with a population of nearly 100 million, is that most populous province in China. Guangdong province has a large demand for agricultural products, low per capita self-sufficiency rate of agricultural products, resulting in frequent fluctuations in agricultural prices. To carry out agricultural economic price dynamic monitoring work, to grasp of the real situation and real-time changes of agricultural economic operation, it can better scientifically and reasonably guide and timely regulate the agricultural production of Guangdong province. This study chose the agricultural products of rice, vegetables, pigs and others to analysis the circulation, consumption price. It can be supported the government to formulate the agriculture policies by researching the agriculture market supply and demand, the agricultural product price monitoring, the agricultural product price warning. It is helpful to promote the development of agricultural scale operation and industrialization, optimize the allocation of resources, shorten the time lag of macro-control of supply and demand of agricultural products. This study can provide effective support for Strengthening the agricultural sector decisionmaking scientific, targeted and timely, improving the level of agricultural products market risk management. The agricultural product price research and application can promote the healthy development of the economy, provide information services and decision support for government departments, scientific research departments, agricultural technology service departments, farmers, agricultural enterprises, cooperatives and other relevant personnel.

Through monitoring the agricultural industry circulation, consumption price dynamic change trend, government departments can real-time access to agricultural products supply and demand data. It is conducive to the government timely adjustment of agricultural policies, increase the support and protection of agriculture, to promote agricultural development to provide timely policy support and public services, to adjust the agricultural industry structure and layout. Agricultural extension and agricultural scientific research departments can compare different regional agricultural products and production technology on the impact of agricultural economic operation, so as to clarify the main direction of agricultural scientific research, scientifically and orderly improve and innovate the agricultural production technology, promote the application of agricultural scientific research achievement. Agricultural producers can be timely aware of the marginal benefits of input factors, understand the different operating performance about the scale of agricultural operation, brand certification, order production. It is conducive to timely adjustment of agricultural production behavior, optimize the production structure, improve the agricultural production and operation efficiency.

Guangdong province of China attaches great importance to agricultural product price monitoring. In 2011, the Guangdong provincial price bureau issued the policies to monitor the price of grain, edible oil, vegetables, pork, chicken and eggs and other six types of agricultural and sideline products market. In recent years, the Guangdong development and reform commission have built "the Guangdong agricultural product price information network". The market and economic information office of the Guangdong agriculture department reports the information of agricultural products price weekly monthly and annual, supplies situation analysis of main agricultural products price. The "Guangdong Price Index Platform" constructed by Guangdong Development and Reform Commission 
includes the monitoring of grain price, the shrimp price, the fruit and vegetable price, the meat price, the fresh agricultural products price, the Chinese herbal medicine price. Now Guangdong pays more attention to monitor the trend of bulk and characteristic agricultural product price and to report regularly early warning and forecast information. In the monitoring analysis such as week, month and annual report, it can operate specific provincial and municipal warning and information release mechanisms Still under construction.

The research institutes involved in the study of agricultural price statistics, monitoring and early warning systems in Guangdong are mainly provincial agriculture-related research institutes, information-related scientific research institutes and some municipal-level agriculture-related inspection or market management agencies. Research focuses on the price information monitoring, early warning modes, early warning systems and mechanisms, with particular attention to the price trend monitoring, index monitoring methods and early warning information system construction. Such as a group of researchers of Guangdong Academy of Agricultural Sciences, and others research agricultural products price fluctuations and market monitoring [1-3]. Another researcher studies the intelligence and early warning mechanisms, reports the number of agricultural products monitoring and early warning of the development of safety Exploration and Analysis [4]. There is a study on the agricultural information monitoring and early warning system in Guangdong Province [5]. One of the researcher and others research institutions such as Guangdong agricultural by-product price warning light management mechanism [6]. A group of researchers studies the application of agricultural price index monitoring [7]. There is also a study in the use of black warning method to the price volatility of the police Indicators, CPI monthly volatility of 1 to 2 times the standard deviation of the average to determine the limit, the establishment of an abnormal price volatility in China's pig market early warning model and start warning [8]. A researcher also reports Guangdong's orange industry sustainable development, to build a circulation Oriented agricultural products market and production early warning system [9]. There is also a study report to build early warning mechanism for aquatic products trade barriers of Guangdong [10-12].

\section{PRICE STATISTICS}

\subsection{Data Source}

Data sources include various types of agricultural statistical yearbooks in China and Guangdong, databases on agricultural price information and indices, and databases on agricultural products networks. It monitored 2 categories including commodity retail price index, wholesale and retail price, 6 varieties and 11 fine items including agricultural products circulation index; The consumer price index of agricultural products consumption index 1 categories, 4 varieties of indicators. The price circulation and consumption data of 8 agricultural products from 7 units including national bureau of statistics, Guangdong bureau of statistics, Guangdong development and reform commission and price monitoring center, Guangdong department of agriculture, Guangdong grain bureau, Guangzhou Jiangnan fruit and vegetable market, Zhengzhou hualiang technology co., ltd. were collected and monitored.

Table 1: Statistical Yearbook Data Source

\begin{tabular}{|c|c|c|}
\hline Statistical Yearbook & Editing unit & content \\
\hline China Statistical Yearbook & National Bureau of Statistics & National agriculture-related economy, price index data \\
\hline China Rural Statistical Yearbook & National Bureau of Statistics & National agricultural economy, agricultural production data \\
\hline Guangdong Statistical Yearbook & Guangdong Bureau of Statistics & Guangdong agriculture-related economy, price index data \\
\hline Guangdong Rural Statistical Yearbook & Guangdong Bureau of Statistics & $\begin{array}{l}\text { Guangdong agriculture-related economy, agricultural } \\
\text { production data }\end{array}$ \\
\hline National Productivity Costs Compilation & $\begin{array}{l}\text { National Development and Reform } \\
\text { Commission Price Division }\end{array}$ & $\begin{array}{c}\text { Production Costs and Income Data of Major Agricultural } \\
\text { Products in China }\end{array}$ \\
\hline $\begin{array}{l}\text { Guangdong agricultural products cost } \\
\text { income compilation }\end{array}$ & $\begin{array}{l}\text { Guangdong Development and Reform } \\
\text { Commission }\end{array}$ & $\begin{array}{l}\text { Guangdong's major agricultural production costs and } \\
\text { revenue data }\end{array}$ \\
\hline
\end{tabular}

This study collects data frequency annual and monthly, daily data. The agricultural circulation data includes price index and price data. The consumption data inclues annual consumer price index data.

Table 2: Data source database

\begin{tabular}{|c|c|c|}
\hline Database & Editing unit & Website \\
\hline China Statistics Network & National Bureau of Statistics & http://www.stats.gov.cn \\
\hline $\begin{array}{c}\text { Guangdong statistical information } \\
\text { network }\end{array}$ & Guangdong Bureau of Statistics & http://www.gdstats.gov.cn/ \\
\hline $\begin{array}{l}\text { Guangdong agricultural product price } \\
\text { information network }\end{array}$ & $\begin{array}{l}\text { Guangdong development and reform } \\
\text { commission and price monitoring center }\end{array}$ & http://www.gdncpjg.cn/ \\
\hline Guangdong price index platform & $\begin{array}{l}\text { Guangdong development and reform } \\
\text { commission and price monitoring center }\end{array}$ & http://www.gdprice.org/ \\
\hline Market information & Guangdong agri & http://www.gdagri.gov.cn/fwpt/scfw/ \\
\hline Market monitoring & Guangdong grain bureau & http://www.gdgrain.gd.gov.cn/xwdt/scjc/ \\
\hline Market analysis & $\begin{array}{l}\text { Jiangnan fruit and vegetable market in } \\
\text { Guangzhou }\end{array}$ & http://www.jnmarket.net/hangqing/list-5.html \\
\hline China grain network & Zhengzhou hualiang technology co., ltd & http://www.cngrain.com/ \\
\hline
\end{tabular}

\subsection{Data Standards and norms}

Annual retail price index data includes rice, meat and meat by-products, fresh vegetables. The daily wholesale prices data of pigs includes the daily price of local hybrid pigs, good hybrid pigs, breeding pigs. The daily wholesale prices data of vegetables includes local Chinese flowering cabbages, lettuce, broccoli. The monthly wholesale prices data of rice includes early indica rice and late indica rice. Annual data of the consumer price index includes rice, meat and poultry products, pork and fresh vegetables.
Circulation Data: The retail price index of commodities collected data from $2002 \sim 2012$, with a point after the decimal point. Pigs wholesale and retail prices collected data from $2010 \sim 2016$, the wholesale price of all kinds of vegetables collected 2010 2015 data, with a point after the decimal point, units: yuan / kg and yuan / kg. Guangdong indica rice and late indica rice Wholesale price collected 1998 2015 data, with two decimal places, unit: yuan / ton. Consumption data: The consumer price index collected data from $2002 \sim 2012$, with one after the decimal point.

Table 3: Data index system

\begin{tabular}{|c|c|c|c|c|}
\hline Primary indicators & Secondary indicators & Thirdary indicators & Forthary indicators & units \\
\hline \multirow{4}{*}{$\begin{array}{l}\text { Agricultural products } \\
\text { circulation indicators }\end{array}$} & Retail Price Index & Rice & & / \\
\hline & & Meat products & & / \\
\hline & & Vegetables & & / \\
\hline & Wholesale and retail & Grain & early indica rice & yuan/to \\
\hline
\end{tabular}




\begin{tabular}{|c|c|c|c|c|}
\hline & prices & & late indica rice & yuan/ton \\
\hline & & Pigs & local hybrid pigs & yuan/half Kg \\
\hline & & & good hybrid pigs & yuan/half Kg \\
\hline & & & breeding pigs & yuan/half Kg \\
\hline & & Vegetables & $\begin{array}{l}\text { local chinese flowering } \\
\text { cabbages }\end{array}$ & yuan/Kg \\
\hline & & & lettuce & yuan/Kg \\
\hline & & & broccoli & yuan/Kg \\
\hline \multirow{4}{*}{$\begin{array}{l}\text { Agricultural consumption } \\
\text { indicators }\end{array}$} & Consumer Price Index & Rice & & / \\
\hline & & Meat Products & & / \\
\hline & & Pork & & / \\
\hline & & Vegetable & & / \\
\hline
\end{tabular}

\subsection{Data Trends}

(1) Agricultural products circulation indicators. Retail price index of commodities, annual data include rice, meat products, fresh vegetables. The annual price series of meat products and fresh vegetables are cyclical, and the annual price fluctuates. There is an uneven change in the annual rice price series.

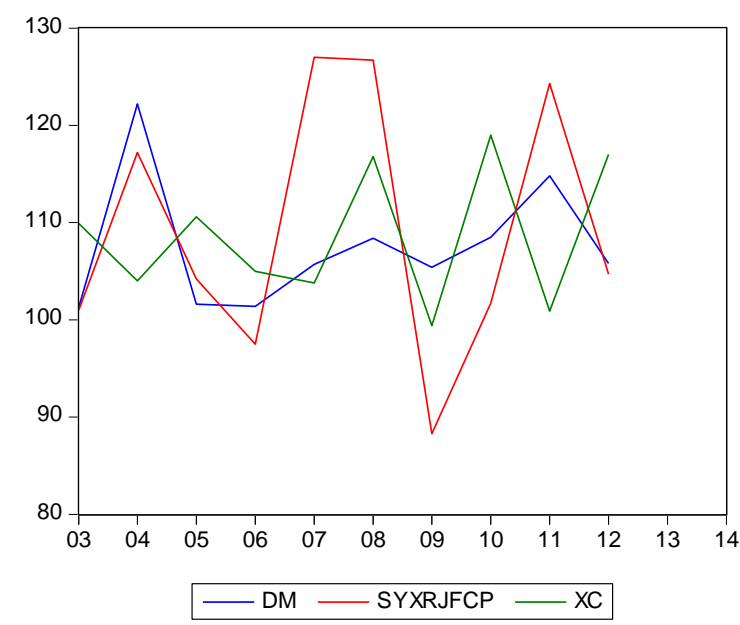

Figure 1: Commodity retail price index annual price series chart DM is rice. SYXRJFCP is Meat products. XC is Vegetables.

Wholesale and retail prices data includes daily data on pig and vegetable prices, and monthly data on rice. The annual and monthly series shows the rice price increase. After 2011, the price of early indica rice fluctuates within the range of 3000 4000 yuan / ton, and the price of late indica rice fluctuates around 5,000 yuan / Ton. The wholesale and retail price time series of pigs are an uneven change. The wholesale price time series of vegetables are extremely unstable.

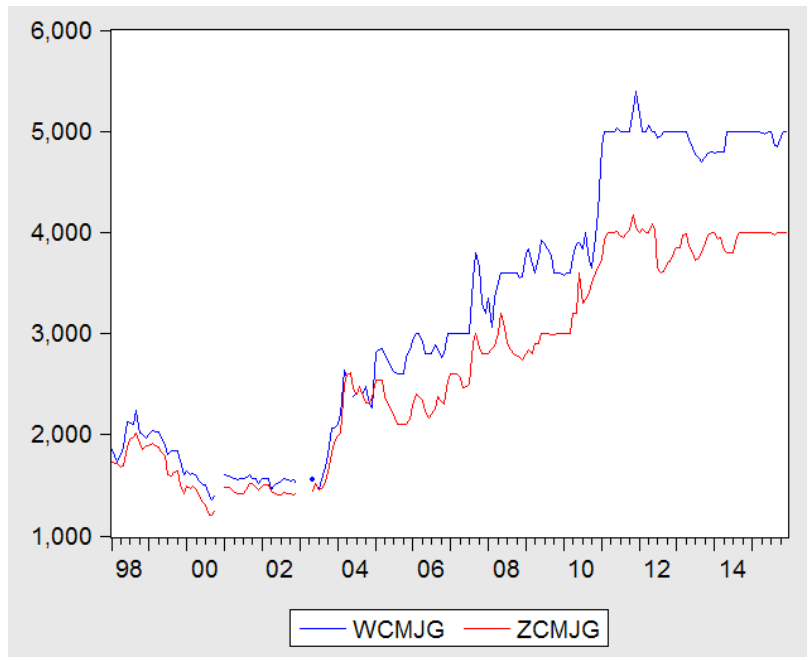

Figure 2: Rice wholesale and retail price monthly time series chart

WCMJG is late indica rice. ZCMJG is early indica rice.

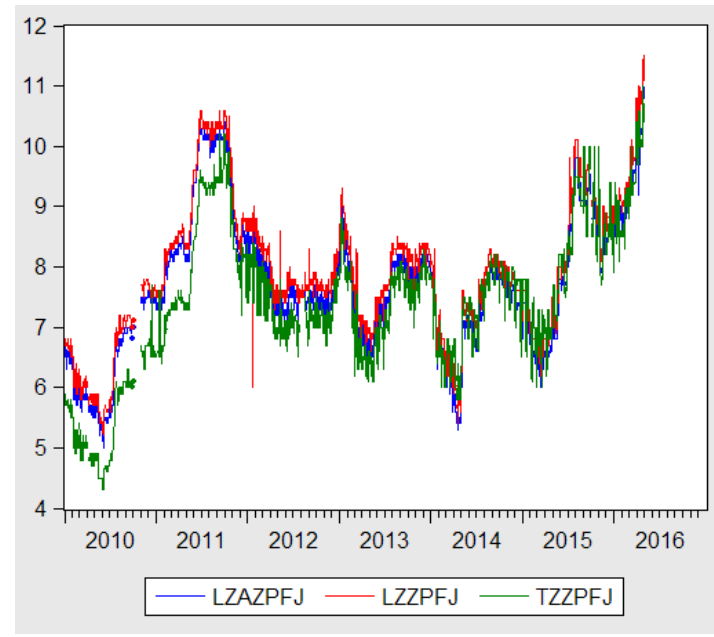

Figure 3: Pigs Wholesale and Retail Prices daily time series chart LZAZPFJ is local hybrid pigs. LZZPFJ is good hybrid pigs. TZZPFJ is breeding pigs.
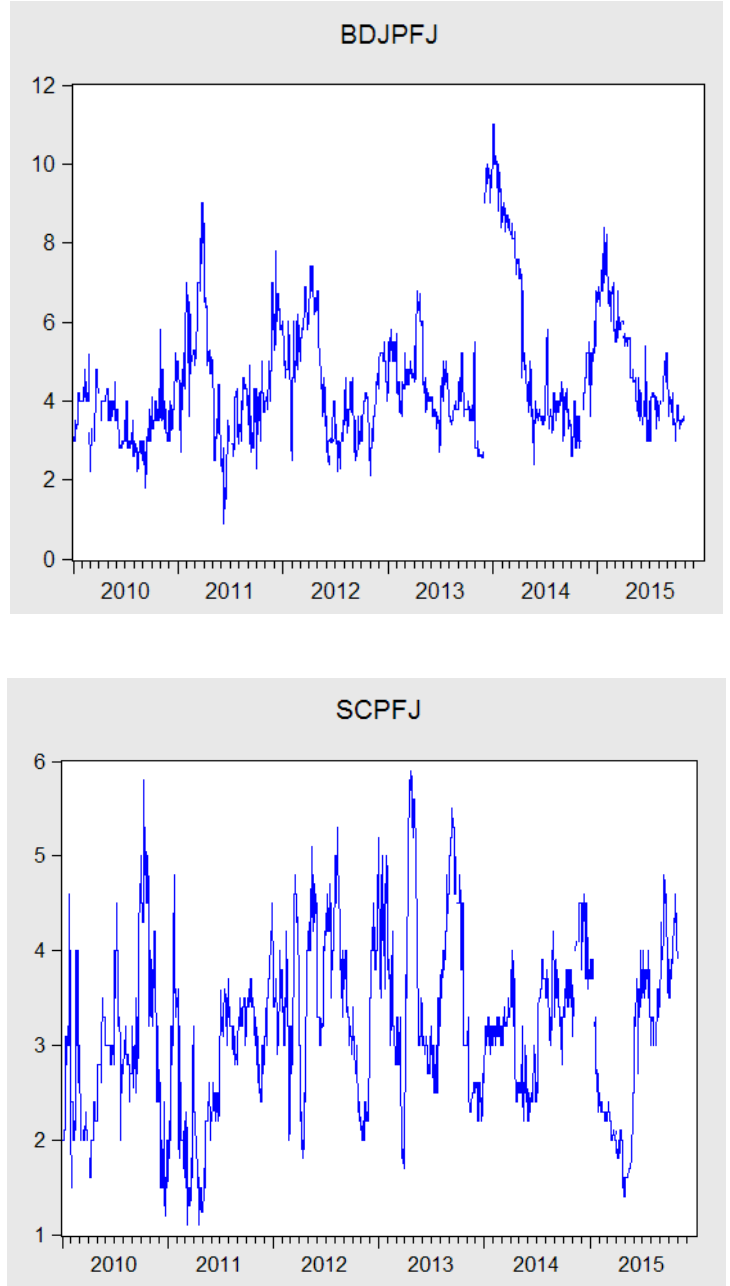


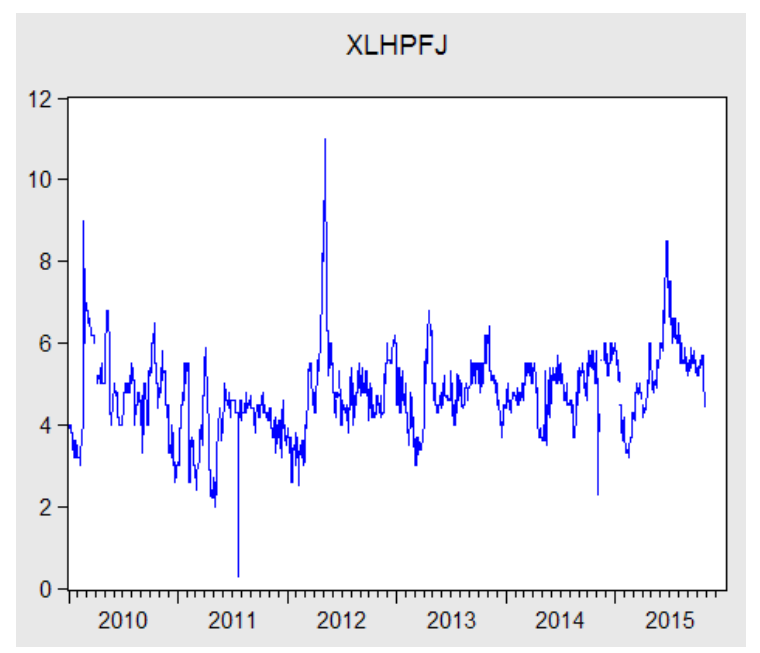

Figure 4: Vegetable Wholesale and Retail Prices daily time series chart $\mathrm{BDJPFJ}$ is local Chinese flowering cabbages. SCPFJ is lettuce. XLHPFJ is broccoli.

(2) Agricultural consumption indicators. Consumer annual price index data, includes rice, meat products, pork and fresh vegetables, in which the consumer price index of rice fluctuates unevenly while the consumer price index of meat, pork and fresh vegetables both have cyclical changes characteristic.

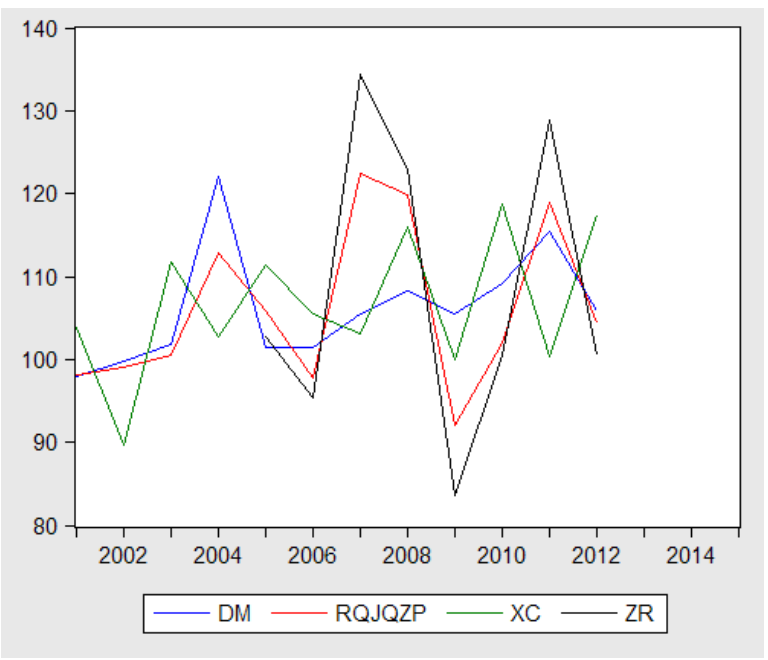

Figure 5: Resident consumer price index annual time series chart $\mathrm{DM}$ is rice. RQJQZP is meat products. XC is vegetable. ZR is pork.

\section{PRICE FORECAST}

\subsection{Methods}

Using statistical software Eviews6.0 analysis of the index data time series trends. Use calculation models includes the exponential smoothing method which includes the double exponential smoothing method and the Holt-Winters seasonal exponential smoothing method for price data trend forecast analysis. Holt-Winters seasonal exponential smoothing method include HW-Non season index method, HW-Additive index method and HW-Multiple index method. The annual agricultural products circulation and consumption index data are fitted by the double exponential smoothing method. The monthly data are fitted and predicted by the HolitWinter seasonal adjustment index smoothing method. The daily data are converted into the monthly data, and fitted and predicted by the HolitWinter seasonal adjustment index smoothed method.

(1) The double exponential smoothing method is the smoothing of an exponential smoothing. It applies to time series with linear trends.

Its prediction formula is:yt $+\mathrm{m}=2+\mathrm{am} / 1-\mathrm{a} \mathbf{X} \mathrm{yt}$ ' $-1+\mathrm{am} / 1-\mathrm{a} \mathbf{\mathrm { X }} \mathrm{yt}=$

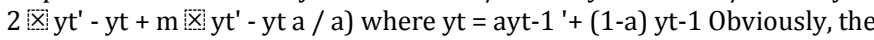
exponential smoothing is a straight line equation with the intercept of (2yt'-yt) Yt) a/(1-a), the independent variable is the forecasted number of days.

The formula is: $\mathrm{St}=\alpha \mathrm{St}+(1-\alpha) \mathrm{St}-1 \mathrm{Yt}+\mathrm{T}=\mathrm{at}+\mathrm{btT}$ at $=2 \mathrm{St}-\mathrm{St} \mathrm{bt}=(\alpha / 1-\alpha)$ (St-St)

(2) The Holt-Winters seasonal exponential smoothing method uses the formula for the adjusted exponential smoothing is:Include trend forecast $(\mathrm{YITt})=$ new forecast $(\mathrm{Yt})+$ trend correction $(\mathrm{Tt})$.There are three steps to exponentially smoothing the trend adjustment: Calculate the simple exponential smoothing forecast $(\mathrm{Yt})$ of period $\mathrm{t}$ using the method described above; calculate the trend.

The formula is: $\mathrm{Tt}=(1-\mathrm{b}) \mathrm{Tt}-1+\mathrm{b}(\mathrm{Yt}-\mathrm{Yt}-1)$

which $\mathrm{Tt}$ = the $\mathrm{t}$-th trend has been smooth; $\mathrm{Tt}-1$ = smoothing trend of the first $\mathrm{t}$ period; $\mathrm{b}=$ trend smoothing factor selected; $\mathrm{Yt}=$ simple exponential smoothing forecast for period $\mathrm{t} ; \mathrm{Yt}-1=$ Smoothing the simple exponent of the previous period t. Calculate trend-adjusted exponential smoothing (YITt). The formula is: YITt $=\mathrm{Yt}+\mathrm{Tt}$.

\subsection{Data calculation}

(1) Circulation indicators of agricultural products: retail price index of commodities include rice, meat products, fresh vegetables. The wholesale and retail prices data include the monthly price data for the early indica rice and late indica rice, the local hybrid pigs, good hybrid pigs and breeding pigs, local Chinese flowering cabbages, lettuce and broccoli.

Using the double exponential smoothing method for data trend fitting and forecasting the retail price index of commodities Retail sales of rice, meat products, fresh vegetables. Compare the data of 2013 and 2014 with the actual value ,the result was showed by Table

Table 4: Double Exponential Method to Predict the Comparison of Retail Price Indices of Commodities in 2013 and2014

\begin{tabular}{ccccc}
\hline Index & Year & Forecast & Actual & Error comparison \\
\hline Rice & 2013 & 104.5298 & 101.0 & $3.49 \%$ \\
Meat Products & 2013 & 103.7714 & 103.0 & $0.75 \%$ \\
Fresh Vegetables & 2013 & 119.5386 & 111.9 & $6.83 \%$ \\
Rice & 2014 & 121.8638 & 101.3 & $20.30 \%$ \\
Meat Products & 2014 & 107.0377 & 100.3 & $6.72 \%$ \\
Fresh Vegetables & 2014 & 106.4827 & 101.2 & $5.22 \%$ \\
\hline
\end{tabular}

Actual value Source: Guangdong Statistical Yearbook

The Holit-Winter seasonally adjusted exponential smoothing method was used to fit the trend of the data and to predict the wholesale and retail prices, include the rice, pigs, vegetables.

Table 5: Holit-Winter seasonally adjusted index forecast early indica rice 2016 monthly wholesale and retail price comparison

\begin{tabular}{cccccccc}
\hline $\begin{array}{c}\mathbf{2 0 1 6} \\
\text { monthly }\end{array}$ & Actual & HW-N & $\begin{array}{c}\text { Error } \\
\text { comparison }\end{array}$ & HW-A & $\begin{array}{c}\text { Error } \\
\text { comparison }\end{array}$ & HW-M & $\begin{array}{c}\text { Error } \\
\text { comparison }\end{array}$ \\
\hline Jan & 5020 & 4020.39 & $-19.91 \%$ & 4049.54 & $-19.33 \%$ & 4075.40 & $-18.82 \%$ \\
Feb & 5050 & 4040.79 & $-19.98 \%$ & 4083.45 & $-19.14 \%$ & 4117.83 & $-18.46 \%$ \\
\hline
\end{tabular}




\begin{tabular}{|c|c|c|c|c|c|c|c|}
\hline Mar & 4975 & 4061.18 & $-18.37 \%$ & 4135.13 & $-16.88 \%$ & 4208.08 & $-15.42 \%$ \\
\hline Apr & 5000 & 4081.58 & $-18.37 \%$ & 4148.68 & $-17.03 \%$ & 4223.15 & $-15.54 \%$ \\
\hline May & 5010 & 4101.97 & $-18.12 \%$ & 4121.21 & $-17.74 \%$ & 4123.97 & $-17.69 \%$ \\
\hline Jun & 5005 & 4122.37 & $-17.63 \%$ & 4125.20 & $-17.58 \%$ & 4112.14 & $-17.84 \%$ \\
\hline Jul & & 4142.76 & & 4035.81 & & 3990.83 & \\
\hline Aug & 4985 & 4163.16 & $-16.49 \%$ & 4078.57 & $-18.18 \%$ & 4043.04 & $-18.90 \%$ \\
\hline Sep & & 4183.55 & & 4108.85 & & 4079.36 & \\
\hline Oct & 5010 & 4203.95 & $-16.09 \%$ & 4120.11 & $-17.76 \%$ & 4083.47 & $-18.49 \%$ \\
\hline Nov & 5085 & 4224.34 & $-16.93 \%$ & 4156.06 & $-18.27 \%$ & 4131.14 & $-18.76 \%$ \\
\hline Dec & 5090 & 4244.74 & $-16.61 \%$ & 4193.20 & $-17.62 \%$ & 4192.65 & $-17.63 \%$ \\
\hline
\end{tabular}

Actual value Source: Guangdong Bureau of Grain statistics

Table 6: Holit-Winter seasonally adjusted index forecast late indica rice 2016 monthly wholesale and retail price comparison

\begin{tabular}{|c|c|c|c|c|c|c|c|}
\hline $\begin{array}{c}2016 \\
\text { monthly }\end{array}$ & Actual & HW-N & $\begin{array}{c}\text { Error } \\
\text { comparison }\end{array}$ & HW-A & $\begin{array}{c}\text { Error } \\
\text { comparison }\end{array}$ & HW-M & $\begin{array}{c}\text { Error } \\
\text { comparison }\end{array}$ \\
\hline Jan & 5020 & 5021.68 & $0.03 \%$ & 5129.70 & $2.19 \%$ & 5198.12 & $3.55 \%$ \\
\hline Feb & 5050 & 5043.35 & $-0.13 \%$ & 5117.55 & $1.34 \%$ & 5178.01 & $2.53 \%$ \\
\hline Mar & 4975 & 5065.03 & $1.81 \%$ & 5130.76 & $3.13 \%$ & 5192.03 & $4.36 \%$ \\
\hline Apr & 5000 & 5086.71 & $1.73 \%$ & 5143.45 & $2.87 \%$ & 5196.71 & $3.93 \%$ \\
\hline May & 5010 & 5108.38 & $1.96 \%$ & 5165.67 & $3.11 \%$ & 5212.62 & $4.04 \%$ \\
\hline Jun & 5005 & 5130.06 & $2.50 \%$ & 5174.03 & $3.38 \%$ & 5213.55 & $4.17 \%$ \\
\hline Jul & & 5151.74 & & 5146.20 & & 5171.55 & \\
\hline Aug & 4985 & 5173.41 & $3.78 \%$ & 5196.12 & $4.24 \%$ & 5254.92 & $5.41 \%$ \\
\hline Sep & & 5195.09 & & 5201.07 & & 5235.95 & \\
\hline Oct & 5010 & 5216.76 & $4.13 \%$ & 5168.71 & $3.17 \%$ & 5180.22 & $3.40 \%$ \\
\hline Nov & 5085 & 5238.44 & $3.02 \%$ & 5184.16 & $1.95 \%$ & 5171.49 & $1.70 \%$ \\
\hline Dec & 5090 & 5260.12 & $3.34 \%$ & 5236.77 & $2.88 \%$ & 5233.23 & $2.81 \%$ \\
\hline
\end{tabular}

Actual value Source: Guangdong Bureau of Grain statistics

Table 7: Holit-Winter seasonally adjusted index forecast the local hybrid pigs half of 2015 monthly wholesale and retail price comparison

\begin{tabular}{|c|c|c|c|c|c|c|c|}
\hline $\begin{array}{c}2015 \\
\text { monthly }\end{array}$ & Actual & HW-N & $\begin{array}{c}\text { Error } \\
\text { comparison }\end{array}$ & HW-A & $\begin{array}{c}\text { Error } \\
\text { comparison }\end{array}$ & HW-M & $\begin{array}{c}\text { Error } \\
\text { comparison }\end{array}$ \\
\hline Jul & 8.43 & 8.04 & $-4.63 \%$ & 8.34 & $-1.07 \%$ & 8.43 & $0.00 \%$ \\
\hline Aug & 8.83 & 8.08 & $-8.49 \%$ & 8.66 & $-1.93 \%$ & 8.83 & $0.00 \%$ \\
\hline Sep & 8.98 & 8.13 & $-9.47 \%$ & 8.8 & $-2.00 \%$ & 8.98 & $0.00 \%$ \\
\hline Oct & 8.86 & 8.17 & $-7.79 \%$ & 8.7 & $-1.81 \%$ & 8.86 & $0.00 \%$ \\
\hline Nov & 8.76 & 8.21 & $-6.28 \%$ & 8.56 & $-2.28 \%$ & 8.76 & $0.00 \%$ \\
\hline Dec & 8.88 & 8.25 & $-7.09 \%$ & 8.66 & $-2.48 \%$ & 8.88 & $0.00 \%$ \\
\hline
\end{tabular}

Actual value Source: Market information of Guangdong agri

Table 8: Holit-Winter seasonally adjusted index forecast the good hybrid pigs half of 2015 monthly wholesale and retail price comparison

\begin{tabular}{|c|c|c|c|c|c|c|c|}
\hline $\begin{array}{c}2015 \\
\text { monthly }\end{array}$ & Actual & HW-N & $\begin{array}{c}\text { Error } \\
\text { comparison }\end{array}$ & HW-A & $\begin{array}{c}\text { Error } \\
\text { comparison }\end{array}$ & HW-M & $\begin{array}{c}\text { Error } \\
\text { comparison }\end{array}$ \\
\hline Jul & 8.8 & 7.83 & $-11.02 \%$ & 8.08 & $-8.18 \%$ & 8.11 & $-7.84 \%$ \\
\hline Aug & 9.4 & 7.85 & $-16.49 \%$ & 8.3 & $-11.70 \%$ & 8.38 & $-10.85 \%$ \\
\hline Sep & 9.3 & 7.88 & $-15.27 \%$ & 8.56 & $-7.96 \%$ & 8.66 & $-6.88 \%$ \\
\hline Oct & 8.8 & 7.91 & $-10.11 \%$ & 8.44 & $-4.09 \%$ & 8.55 & $-2.84 \%$ \\
\hline Nov & 8.5 & 7.94 & $-6.59 \%$ & 8.26 & $-2.82 \%$ & 8.42 & $-0.94 \%$ \\
\hline Dec & 8.6 & 7.96 & $-7.44 \%$ & 8.28 & $-3.72 \%$ & 8.44 & $-1.86 \%$ \\
\hline
\end{tabular}

Actual value Source: Market information of Guangdong agri

Table 9: Holit-Winter seasonally adjusted index forecast the breeding pigs half of 2015 monthly wholesale and retail price comparison

\begin{tabular}{|c|c|c|c|c|c|c|c|}
\hline $\begin{array}{c}2015 \\
\text { monthly }\end{array}$ & Actual & HW-N & $\begin{array}{c}\text { Error } \\
\text { comparison }\end{array}$ & HW-A & $\begin{array}{c}\text { Error } \\
\text { comparison }\end{array}$ & HW-M & $\begin{array}{c}\text { Error } \\
\text { comparison }\end{array}$ \\
\hline Jul & 9.1 & 8.03 & $-11.76 \%$ & 8.4 & $-7.69 \%$ & 8.40 & $-7.69 \%$ \\
\hline Aug & 9.7 & 8.07 & $-16.80 \%$ & 8.68 & $-10.52 \%$ & 8.73 & $-10.00 \%$ \\
\hline Sep & 9.6 & 8.10 & $-15.63 \%$ & 9.04 & $-5.83 \%$ & 9.17 & $-4.48 \%$ \\
\hline Oct & 9.1 & 8.14 & $-10.55 \%$ & 9.14 & $0.44 \%$ & 9.27 & $1.87 \%$ \\
\hline Nov & 8.7 & 8.17 & $-6.09 \%$ & 8.98 & $3.22 \%$ & 9.10 & $4.60 \%$ \\
\hline Dec & 8.9 & 8.21 & $-7.75 \%$ & 8.82 & $-0.90 \%$ & 8.99 & $1.01 \%$ \\
\hline
\end{tabular}

Actual value Source: Market information of Guangdong agri

Table 10: Holit-Winter seasonally adjusted index forecast local chinese flowering cabbages 2015 monthly wholesale and retail price comparison

\begin{tabular}{|c|c|c|c|c|c|c|c|}
\hline $\begin{array}{c}2015 \\
\text { monthly }\end{array}$ & Actual & HW-N & $\begin{array}{c}\text { Error } \\
\text { comparison }\end{array}$ & HW-A & $\begin{array}{c}\text { Error } \\
\text { comparison }\end{array}$ & HW-M & $\begin{array}{c}\text { Error } \\
\text { comparison }\end{array}$ \\
\hline Jan & 3.0 & 4.23 & $41.00 \%$ & 4.25 & $41.67 \%$ & 4.38 & $46.00 \%$ \\
\hline Feb & 2.2 & 4.34 & $97.27 \%$ & 3.73 & $69.55 \%$ & 3.70 & $68.18 \%$ \\
\hline Mar & 5.0 & 4.46 & $-10.80 \%$ & 4.55 & $-9.00 \%$ & 4.66 & $-6.80 \%$ \\
\hline Apr & 3.3 & 4.57 & $38.48 \%$ & 3.89 & $17.88 \%$ & 3.82 & $15.76 \%$ \\
\hline May & 4.0 & 4.68 & $17.00 \%$ & 4.65 & $16.25 \%$ & 4.81 & $20.25 \%$ \\
\hline
\end{tabular}

Cite The Article: Li Dan-Xia , Wan Zhong (2018). Statistics, Monitoring And Early Warning Of Agricultural Products Circulation And Consumption Price In Guan gdong Province Of China . Topics in Economics, Business and Management, 2(1) : 52-59. 
Topics in Economics, Business and Management (EBM) 2(1) (2018) 52-59

\begin{tabular}{|c|c|c|c|c|c|c|c|}
\hline Jun & 5.3 & 4.80 & $-9.43 \%$ & 4.95 & $-6.60 \%$ & 5.08 & $-4.15 \%$ \\
\hline Jul & 4.4 & 4.91 & $11.59 \%$ & 6.41 & $45.68 \%$ & 6.79 & $54.32 \%$ \\
\hline Aug & 4.8 & 5.02 & $4.58 \%$ & 5.75 & $19.79 \%$ & 6.00 & $25.00 \%$ \\
\hline Sep & 6.8 & 5.14 & $-24.41 \%$ & 5.77 & $-15.15 \%$ & 5.96 & $-12.35 \%$ \\
\hline Oct & 6.5 & 5.25 & $-19.23 \%$ & 4.55 & $-30.00 \%$ & 4.65 & $-28.46 \%$ \\
\hline Nov & 4.2 & 5.36 & $27.62 \%$ & 3.85 & $-8.33 \%$ & 3.81 & $-9.29 \%$ \\
\hline Dec & 5.9 & 5.48 & $-7.12 \%$ & 4.25 & $-27.97 \%$ & 4.30 & $-27.12 \%$ \\
\hline
\end{tabular}

Actual value Source: Market information of Jiangnan fruit and vegetable market in Guangzhou

Table 11: Holit-Winter seasonally adjusted index forecast lettuce 2015 monthly wholesale and retail price comparison

\begin{tabular}{|c|c|c|c|c|c|c|c|}
\hline $\begin{array}{c}2015 \\
\text { monthly }\end{array}$ & Actual & HW-N & $\begin{array}{c}\text { Error } \\
\text { comparison }\end{array}$ & HW-A & $\begin{array}{c}\text { Error } \\
\text { comparison }\end{array}$ & HW-M & $\begin{array}{c}\text { Error } \\
\text { comparison }\end{array}$ \\
\hline Jan & 3.3 & 3.78 & $14.55 \%$ & 3.70 & $12.12 \%$ & 3.67 & $11.21 \%$ \\
\hline Feb & 2.3 & 3.81 & $65.65 \%$ & 3.36 & $46.09 \%$ & 3.30 & $43.48 \%$ \\
\hline Mar & 2.2 & 3.84 & $74.55 \%$ & 3.04 & $38.18 \%$ & 2.92 & $32.73 \%$ \\
\hline Apr & 1.8 & 3.87 & $115.00 \%$ & 3.26 & $81.11 \%$ & 3.12 & $73.33 \%$ \\
\hline May & 1.8 & 3.90 & $116.67 \%$ & 3.58 & $98.89 \%$ & 3.49 & $93.89 \%$ \\
\hline Jun & 3.4 & 3.93 & $15.59 \%$ & 3.20 & $-5.88 \%$ & 3.13 & $-7.94 \%$ \\
\hline Jul & 3.6 & 3.96 & $10.00 \%$ & 3.82 & $6.11 \%$ & 3.84 & $6.67 \%$ \\
\hline Aug & 3.3 & 3.99 & $20.91 \%$ & 3.92 & $18.79 \%$ & 3.90 & $18.18 \%$ \\
\hline Sep & 4.1 & 4.02 & $-1.95 \%$ & 4.04 & $-1.46 \%$ & 4.05 & $-1.22 \%$ \\
\hline Oct & 4.1 & 4.05 & $-1.22 \%$ & 4.02 & $-1.95 \%$ & 4.10 & $0.00 \%$ \\
\hline Nov & 2.7 & 4.08 & $51.11 \%$ & 3.38 & $25.19 \%$ & 3.37 & $24.81 \%$ \\
\hline Dec & 3.9 & 4.11 & $5.38 \%$ & 3.60 & $-7.69 \%$ & 3.58 & $-8.21 \%$ \\
\hline
\end{tabular}

Actual value Source: Market information of Jiangnan fruit and vegetable market in Guangzhou

Table 12: Holit-Winter seasonally adjusted index forecast broccoli 2015 monthly wholesale and retail price comparison

\begin{tabular}{|c|c|c|c|c|c|c|c|}
\hline $\begin{array}{c}2015 \\
\text { monthly }\end{array}$ & Actual & HW-N & $\begin{array}{c}\text { Error } \\
\text { comparison }\end{array}$ & HW-A & $\begin{array}{c}\text { Error } \\
\text { comparison }\end{array}$ & HW-M & $\begin{array}{c}\text { Error } \\
\text { comparison }\end{array}$ \\
\hline Jan & 4.7 & 5.50 & $17.02 \%$ & 4.66 & $-0.85 \%$ & 4.66 & $-0.85 \%$ \\
\hline Feb & 3.5 & 5.52 & $57.71 \%$ & 4.68 & $33.71 \%$ & 4.65 & $32.86 \%$ \\
\hline Mar & 4.6 & 5.55 & $20.65 \%$ & 5.16 & $12.17 \%$ & 5.15 & $11.96 \%$ \\
\hline Apr & 4.8 & 5.57 & $16.04 \%$ & 5.50 & $14.58 \%$ & 5.53 & $15.21 \%$ \\
\hline May & 5.2 & 5.59 & $7.50 \%$ & 5.44 & $4.62 \%$ & 5.45 & $4.81 \%$ \\
\hline Jun & 7.0 & 5.62 & $-19.71 \%$ & 5.20 & $-25.71 \%$ & 5.24 & $-25.14 \%$ \\
\hline Jul & 6.4 & 5.64 & $-11.88 \%$ & 5.08 & $-20.63 \%$ & 5.10 & $-20.31 \%$ \\
\hline Aug & 5.6 & 5.66 & $1.07 \%$ & 5.10 & $-8.93 \%$ & 5.13 & $-8.39 \%$ \\
\hline Sep & 5.5 & 5.69 & $3.45 \%$ & 5.30 & $-3.64 \%$ & 5.34 & $-2.91 \%$ \\
\hline Oct & 5.2 & 5.71 & $9.81 \%$ & 5.52 & $6.15 \%$ & 5.57 & $7.12 \%$ \\
\hline Nov & 4.3 & 5.73 & $33.26 \%$ & 5.34 & $24.19 \%$ & 5.37 & $24.88 \%$ \\
\hline Dec & 4.4 & 5.76 & $30.91 \%$ & 5.06 & $15.00 \%$ & 5.05 & $14.77 \%$ \\
\hline
\end{tabular}

Actual value Source: Market information of Jiangnan fruit and vegetable market in Guangzhou

(2) Consumption indicators of agricultural products: the annual consumer price index data include rice, meat products, pork and fresh vegetables.

Table 13: Double Exponential Method to Predict Produce Index of Agricultural Products in 2013, 2014 and 2015

\begin{tabular}{|c|c|c|c|c|}
\hline Index & Year & Forecast & Actual & Error comparison \\
\hline Rice & \multirow{4}{*}{2013} & 115.71 & 100.7 & $-14.91 \%$ \\
\hline Meat products & & 110.64 & 102.0 & $-8.47 \%$ \\
\hline Pork & & 115.57 & 99.6 & $-16.03 \%$ \\
\hline Fresh vegetables & & 119.08 & 111.4 & $-6.89 \%$ \\
\hline Rice & \multirow{4}{*}{2014} & 116.94 & 101.4 & $-15.33 \%$ \\
\hline Meat products & & 111.50 & 103.3 & $-7.94 \%$ \\
\hline Pork & & 118.46 & 98.8 & $-19.90 \%$ \\
\hline Fresh vegetables & & 120.87 & 101.0 & $-19.67 \%$ \\
\hline Rice & \multirow{4}{*}{2015} & 118.17 & 101.1 & $-14.45 \%$ \\
\hline Meat products & & 112.37 & 106.0 & $-5.67 \%$ \\
\hline Pork & & 121.34 & 106.9 & $-11.90 \%$ \\
\hline Fresh vegetables & & 122.67 & 107.8 & $-12.12 \%$ \\
\hline
\end{tabular}

Actual value Source: Guangdong Statistical Yearbook

\subsection{Data Analysis}

Circulation indicators of agricultural products: Commodity retail price index was forecasted by the double exponential smoothing method for annual data, which is the forecast error in the range of $0.75 \% \sim 20.30 \%$. Wholesale and retail prices of agricultural products was forecasted by Holit-Winter season adjustment index smoothing method for monthly data, which the forecast error values are very difference for each product.
Rice wholesale and retail price error value is less than $25 \%$. Pigs wholesale price error value is less than $25 \%$. Vegetable wholesale price error value is about $100 \%$. Consumption indicators of agricultural products: Consumer Price Index was forecasted by the double exponential smoothing method for annual data, the absolute value of the forecast error in the range of $5.67 \% \sim 19.90 \%$. 
Topics in Economics, Business and Management (EBM) 2(1) (2018) 52-59

Table 14: Agricultural products circulation and consumption indicators error value, alert level, early warning range

\begin{tabular}{|c|c|c|c|c|c|c|c|c|c|c|}
\hline $\begin{array}{c}\text { Primary } \\
\text { indicators }\end{array}$ & $\begin{array}{l}\text { Secondary } \\
\text { indicators }\end{array}$ & $\begin{array}{l}\text { Thirdary } \\
\text { indicators }\end{array}$ & $\begin{array}{l}\text { Forthary } \\
\text { indicators }\end{array}$ & $\begin{array}{l}\text { Forecast } \\
\text { Modele }\end{array}$ & $\begin{array}{l}\text { Min } \\
\text { error } \\
\text { value }\end{array}$ & $\begin{array}{c}\text { Max } \\
\text { error } \\
\text { value }\end{array}$ & Units & $\begin{array}{c}\text { Min } \\
\text { alert }\end{array}$ & $\begin{array}{c}\text { Max } \\
\text { alert }\end{array}$ & $\begin{array}{c}\text { Early } \\
\text { warning } \\
\text { Range }( \pm)\end{array}$ \\
\hline \multirow{27}{*}{$\begin{array}{l}\text { Agricultural } \\
\text { products } \\
\text { circulation } \\
\text { indicators }\end{array}$} & \multirow[t]{3}{*}{$\begin{array}{l}\text { Retail Price } \\
\text { Index }\end{array}$} & Rice & & \multirow{3}{*}{$\begin{array}{c}\text { double } \\
\text { exponential } \\
\text { smoothing } \\
\text { method }\end{array}$} & $3.49 \%$ & $20.30 \%$ & / & 122.2 & 100.3 & $25 \%$ \\
\hline & & $\begin{array}{l}\text { Meat } \\
\text { products }\end{array}$ & & & $0.75 \%$ & $6.72 \%$ & / & 124.3 & 100.3 & $10 \%$ \\
\hline & & Vegetables & & & $5.22 \%$ & $6.83 \%$ & / & 117 & 100.9 & $10 \%$ \\
\hline & \multirow{24}{*}{$\begin{array}{l}\text { Wholesale } \\
\text { and retail } \\
\text { prices }\end{array}$} & \multirow[t]{6}{*}{ Grain } & \multirow{3}{*}{$\begin{array}{c}\text { early } \\
\text { indica rice }\end{array}$} & $\mathrm{HW}-\mathrm{N}$ & $16.09 \%$ & $19.98 \%$ & \multirow{3}{*}{ yuan/ton } & \multirow{3}{*}{5090} & \multirow{3}{*}{3000} & \multirow{3}{*}{$25 \%$} \\
\hline & & & & HW-A & $17.03 \%$ & $19.33 \%$ & & & & \\
\hline & & & & HW-M & $15.42 \%$ & $18.90 \%$ & & & & \\
\hline & & & \multirow{3}{*}{$\begin{array}{l}\text { late indica } \\
\text { rice }\end{array}$} & $\mathrm{HW}-\mathrm{N}$ & $0.03 \%$ & $4.13 \%$ & \multirow{3}{*}{ yuan/ton } & \multirow{3}{*}{5090} & \multirow{3}{*}{3580} & \multirow{3}{*}{$10 \%$} \\
\hline & & & & HW-A & $1.34 \%$ & $4.24 \%$ & & & & \\
\hline & & & & HW-M & $1.70 \%$ & $5.41 \%$ & & & & \\
\hline & & \multirow[t]{9}{*}{ Pigs } & \multirow{3}{*}{$\begin{array}{l}\text { local } \\
\text { hybrid } \\
\text { pigs }\end{array}$} & $\mathrm{HW}-\mathrm{N}$ & $4.63 \%$ & $9.47 \%$ & \multirow{3}{*}{ yuan/Kg } & \multirow{3}{*}{22} & \multirow{3}{*}{8.6} & \multirow{3}{*}{$10 \%$} \\
\hline & & & & $\mathrm{HW}-\mathrm{A}$ & $1.07 \%$ & $2.48 \%$ & & & & \\
\hline & & & & HW-M & $0.00 \%$ & $0.00 \%$ & & & & \\
\hline & & & \multirow{3}{*}{$\begin{array}{l}\text { good } \\
\text { hybrid } \\
\text { pigs }\end{array}$} & HW-N & $6.59 \%$ & $16.49 \%$ & \multirow{3}{*}{ yuan/Kg } & \multirow{3}{*}{22.4} & \multirow{3}{*}{1.4} & \multirow{3}{*}{$25 \%$} \\
\hline & & & & HW-A & $2.82 \%$ & $11.70 \%$ & & & & \\
\hline & & & & HW-M & $0.94 \%$ & $10.85 \%$ & & & & \\
\hline & & & \multirow{3}{*}{$\begin{array}{l}\text { breeding } \\
\text { pigs }\end{array}$} & HW-N & $6.09 \%$ & $16.80 \%$ & \multirow{3}{*}{ yuan/Kg } & & & \\
\hline & & & & HW-A & $0.44 \%$ & $10.52 \%$ & & 23 & 10.4 & $25 \%$ \\
\hline & & & & HW-M & $1.01 \%$ & $10.00 \%$ & & & & \\
\hline & & Vegetables & & $\mathrm{HW}-\mathrm{N}$ & $4.58 \%$ & $97.27 \%$ & & & & \\
\hline & & & $\begin{array}{l}\text { chinese } \\
\text { flowering }\end{array}$ & HW-A & $6.60 \%$ & $69.55 \%$ & $\begin{array}{c}\text { yuan/half } \\
\text { Ka }\end{array}$ & 10 & 1.1 & $100 \%$ \\
\hline & & & cabbages & HW-M & $4.15 \%$ & $68.18 \%$ & & & & \\
\hline & & & lettuce & $\mathrm{HW}-\mathrm{N}$ & $1.22 \%$ & $116.67 \%$ & & & & \\
\hline & & & & HW-A & $1.46 \%$ & $98.89 \%$ & yuan/half & 5.9 & 1.3 & $100 \%$ \\
\hline & & & & HW-M & $0.00 \%$ & $93.89 \%$ & & & & \\
\hline & & & broccoli & $\mathrm{HW}-\mathrm{N}$ & $1.07 \%$ & $57.71 \%$ & yuan/half & & & \\
\hline & & & & HW-A & $0.85 \%$ & $33.71 \%$ & $\mathrm{Kg}$ & 11 & 2 & $75 \%$ \\
\hline & & & & HW-M & $0.85 \%$ & $32.86 \%$ & & & & \\
\hline $\begin{array}{l}\text { Agricultural } \\
\text { consumption }\end{array}$ & $\begin{array}{l}\text { Consumer } \\
\text { Price Index }\end{array}$ & Rice & & $\begin{array}{c}\text { double } \\
\text { exponential }\end{array}$ & $0.21 \%$ & $74.83 \%$ & / & 115.4 & 101.1 & $25 \%$ \\
\hline itors & & $\begin{array}{c}\text { Meat } \\
\text { Products }\end{array}$ & & $\begin{array}{l}\text { smoothing } \\
\text { method }\end{array}$ & $1.40 \%$ & $52.07 \%$ & / & 118.9 & 102 & $10 \%$ \\
\hline & & Pork & & & $0.60 \%$ & $56.18 \%$ & / & 128.8 & 98.8 & $25 \%$ \\
\hline & & Vegetable & & & $3.36 \%$ & $47.87 \%$ & I & 117.5 & 100.3 & $25 \%$ \\
\hline
\end{tabular}

\section{PRICE WARNING}

\subsection{Early warning cord set}

Specific Early warning indicators were showed by table.

Table 15: Early warning indicators

\begin{tabular}{ccc}
\hline Alert limit & Alert level & Market characteristics \\
\hline$(-\infty,-2 \delta)$ & Negative seriously & Trend down, greatest volatility, the market is too cold (abnormal) \\
{$[-2 \delta,-\delta)$} & Negative lightly & Trend down, greater volatility, the market is cold (abnormal) \\
{$[-\delta,+\delta]$} & No alert & Light volatility, the market is basically balanced (normal) \\
$(+\delta,+2 \delta]$ & Positive lightly & Trend up, greater volatility, the market is hot (abnormal) \\
$(+2 \delta,+\infty)$ & Positive seriously & Trend up, greatest volatility, the market is hot (abnormal) \\
\hline
\end{tabular}

\subsection{Early warning value}

The characteristics of each indicator series data are different. For example, the retail price index of commodities, wholesale and retail prices of vegetables, and consumer price index series have the characteristics of periodic fluctuation. The rice, pigs wholesale and retail price data have time-series features. The warning value has the reference meaning between the highest value and the lowest value from 2010 to the present, which has the characteristics of periodic fluctuation. The warning value has the reference meaning in the error range of forecast value for the characteristic sequence of time trend. Early warning value, warning forecast range see table.

\section{CONCLUSIONS AND RECOMMENDATIONS}

\subsection{Conclusions}

The study collects statistics and monitor the prices of rice, pigs and vegetables, established basic wholesale and retail agricultural products price database of Guangdong agriculture. Through using the exponential smoothing model, the error value of the retail price index of the commodity is within $25 \%$. The error value of the wholesale and retail price index of rice and pigs is within $25 \%$. Vegetable prices error value fluctuated between $50 \%$ and $100 \%$, and the forecasted value fluctuates slightly. Except the wholesale and retail prices of vegetable, all the forecast error value of retail price index, wholesale and retail prices, consumer price index are within $25 \%$. Especially the retail price index of meat products and vegetables, the wholesale and retail prices of late indica rice and local hybrid pigs, the consumer price index of meat products, which 
It means the forecast of the exponential smoothing model quitly exact. The wholesale and retail prices of vegetable may be affected by different plant origin inside and outside Guangdong Province, season and climate change, resulting a large error between the forecast value and the actual value. And according to the forecast value, we set double Early warning cord. One cord is settled between the highest value and the lowest value from 2010 to the present. If the forecast value exceeds the historical value, the alert alarm is responded. The other cord is settled within the early warning range. If the forecast value exceeds the latest value early warning range, the alert alarm is responded.

What we study the agricultural product circulation and consumption price statistics, agricultural product price monitoring and early warning, is used in the research of "Guangdong provincial research institutions reform and innovation project", and "Guangdong Academy of Agricultural Sciences Institute of Agricultural Economics and Rural Development Fund Project" to analysis the development and the change of the Guangdong province agriculture products price.

\subsection{Recommendations}

In the process of data collection and monitoring, this study found that the existing agricultural product price statistics have different calibers, and the names, regions, standards, and statistics time. It is difficult to accurately forecast the price of agricultural products by the different data. Such as: China Food Network, the rice name and unit standard are quitly different from the different plant regions, and the data count at different times. The China Food Network data from Guangdong central reserve grain Zhaoqing library, which the indica rice purchase no quotation after 2016. The China Food Network data from Shenzhen library, which the statistics standard is only one column "rice" without the comparison standard of early indica rice and late indica rice. Such as: Guangdong price index platform, the standard quotations of various rice companies vary. The Guangdong price index platform data from Dongguan Changping grain and oil market quoted rice name: pearl rice, silk seedlings rice, rice from other countries. The Guangdong price index platform data from XingLi Trade City agriculture market quoted rice name: pearl rice, late highquality rice, Late rice, etc. The Guangdong price index platform data from Guangzhou Railway's Sanyanqiao Grain Market quoted rice name: Xiantao fragrant rice, snowflake incense and sticky, etc. The statistical agriculture products name and unit is quietly different from different network, data platform, resulting to count and forecast the agricultural product price difficulty.

The problems in the agricultural early warning system in Guangdong mainly has the following aspects. First, although the quality and safety monitoring system for agricultural products has been gradually established and the information publicity system has been implemented, the monitoring and early warning of quality and safety needs to be developed and perfected. The early warning mechanism for quality and safety of agricultural products has not yet been formed, Second, the Guangdong Development and Reform Commission and its Price Bureau, Price Monitoring Center in 2011 to build a key agricultural market price warning mechanism, the Guangdong Provincial Department of Agriculture has also established a mechanism for publicity of agricultural product price information, Establish a market system for agricultural product supply and price release, but the status of specific agricultural product price information warnings and the release of indicator information have yet to be improved. The establishment of the Office of the indicators and the monitoring price system has not yet formed a unified, public information convergence has not yet been unified, has not yet been able to make the early warning of price fluctuations information disclosure; Third, Guangdong Agricultural Early Warning System of agricultural prices, agricultural product quality and safety monitoring and early warning has been gradually Construction molding, price and quality safety monitoring is still limited to the Guangdong Province, the domestic agricultural products have become increasingly inter-provincial production and circulation of agricultural products in Guangdong into or through the price of quality and safety monitoring and early warning, Guangdong still need We will form monitoring and early warning publicity links with the prices of agricultural products such as the Ministry of Agriculture and the National Development and Reform Commission, and the quality and safety of agricultural products.

According to the research, Guangdong province need to construct a early warning agriculture price system which can be used in different improve the construction of a provincial-level system for monitoring agricultural product price information and monitoring the quality and safety of agricultural products, improve public information collection standards, collection channels and information disclosure, and timely link up the construction of a national system. We must innovate and develop agricultural early-warning mechanisms and technologies and continue to develop agricultural early-warning systems. It is necessary to establish Guangdong application-type warning information publicity and publicity system for expanding the role of agricultural early warning, innovating development of service mechanisms, convergence futures market, government regulation and other needs. It is necessary to research and innovation based on the network, big data mining and other agricultural industry, agricultural prices, agricultural real-time production / sales of agricultural warning system.

\section{ABOUT THE AUTHORS}

Li Danxia: the first author, correspondent, assistant researcher, mainly engaged in agricultural economic policy research, agricultural industry economic research; Address: No.31, Jinying Road, Wushan Street, Tianhe District, Guangzhou City, Guangdong Province, China. Postcodes:510640. Telephone Number: +86 13798172279,+0086 020-38319952.Email:625005883@qq.com.

Wan Zhong: Researcher, Deputy Director, vice director of Institute of Agricultural Economy and Rural Development, Guangdong Academy of Agricultural Sciences

\section{REFERENCES}

[1] Luo, J., Fang, W., Lin, W.J., Wan, Z. 2012. Review of agricultural product price volatility and intelligence early warning. Journal of Guangdong Agricultural Sciences, 11, 212-214 + 223 .

[2] Zheng, S.F., Cheng, Y.L., Lin, W.J., Ma, W., Li, Z. 2012. Review of China's agricultural product market monitoring and early warning. Journal of Guangdong Agricultural Sciences, 23, 228-231.

[3] Kang, Y.Z., Fang, W., Lin, W.J. 2014. Early Warning Analysis of Abnormal Volatility Information of Important Agricultural Products in China. Journal of Guangdong Agricultural Sciences, 07, 200-203.

[4] Huang, H.X., Li, Z., Zheng, Y.L. 2014. Probing into the Development of Agricultural Products Safety Monitoring and Early Warning. Journal of Agricultural Outlook, 03, 50-53.

[5] Liu, X.K., Lin, L.Y., Huang, H.X., He, Y.Q. 2014. Discussion on Monitoring and Early Warning System of Agricultural Information in Guangdong Province [J] Journal of Modern Agricultural Science and Technology, 20, 326-327

[6] Su, W.G., He, X.C. 2011. Guangdong agricultural by-product price warning signal management mechanism. Journal of Market Economy and Price, 11, 23-24.

[7] Zhu, S.H., Chen, D.H. 2011. Innovative price monitoring method based on index resources - A preliminary study on price index based on correlation analysis. Journal of Market Economics and Value, 12, 32-35.

[8] Zhu, S.H., Chen, D.H. 2011. Using price index resources to innovate price monitoring methods - Based on the application of Jiangnan fruits and vegetables index resource in Guangdong price monitoring Journal of Price Theory and Practice, 11, 56-57.

[9] Chen, Z.Y. 2011. China tropical fruit - litchi, longan processing industry monitoring analysis and early warning. Journal of Agricultural Engineering Technology - Agro-industry, 10, 21-29.

[10] Kang, Y.Z., Zheng, Y.L., Lin, W.J., Fang, W. 2016. Early warning of abnormal fluctuations in pig market in China and its countermeasures. Journal of South Rural, 01, 46-49.

[11] Wang, X.Q. 2009. Research on the Construction of Agricultural Products Market and Production Early Warning System Based on Circulation-oriented Model - Taking the Sustainable Development of Citrus Industry in Guangdong as an Example. Journal of Guangdong Science and Technology,2009,11, 79-80.

[12] Gao, W.X. 2010. Establishing an Early Warning Mechanism for Aquatic Products Trade Barriers - A Case Study of Guangdong Journal of Jiangdong Ocean University, 02, 51-56. 